\title{
Pressure-dependent water absorption cross sections for exoplanets and other atmospheres.
}

\author{
Emma J. Barton, C. Hill, Sergei N. Yurchenko, Jonathan Tennyson \\ Department of Physics and Astronomy, University College London, London, WC1E \\ $6 B T, U K$
}

Anna S. Dudaryonok, Nina N. Lavrentieva

V. E. Zuev Institute of Atmospheric Optics SB RAS, 1, Sq. Academician Zuev, 634021

Tomsk, Russia.

\begin{abstract}
Many atmospheres (cool stars, brown dwarfs, giant planets, extrasolar planets) are predominately composed of molecular hydrogen and helium. $\mathrm{H}_{2}{ }^{16} \mathrm{O}$ is one of the best measured molecules in extrasolar planetary atmospheres to date and a major compound in the atmospheres of brown-dwarfs and oxygen-rich cool stars, yet the scope of experimental and theoretical studies on the pressure broadening of water vapour lines by collision with hydrogen and helium remains limited.

Theoretical $\mathrm{H}_{2}$ - and He-broadening parameters of water vapour lines (rotational quantum number $J$ up to 50) are obtained for temperatures in the range 300 - $2000 \mathrm{~K}$. Two approaches for calculation of line widths were used: (i) the averaged energy difference method and (ii) the empirical expression for $J^{\prime} J^{\prime \prime}$-dependence.
\end{abstract}

Voigt profiles based on these widths and the BT2 line list are used to generate high resolution $\left(\Delta \tilde{\nu}=0.01 \mathrm{~cm}^{-1}\right)$ pressure broadened cross sections 
for a fixed range of temperatures and pressures between 300 - $2000 \mathrm{~K}$ and 0.001 - 10 bar. An interpolation procedure which can be used to determine cross sections at intermediate temperature and pressure is described. Pressure broadening parameters and cross sections are presented in new ExoMol format.

Key words:

Water, Cross sections, Pressure broadening, Atmospheres, Extrasolar planets, BT2, Composition

\section{Introduction}

The ExoMol project aims to provide comprehensive line lists of molecular transitions appropriate for molecules in hot atmospheres such as those found in exoplanets, brown dwarfs and cool stars [1]. Until recently the ExoMol database did not include any information on the pressure broadening of molecular lines despite the known importance of pressure effects in these environments [2]. The scope of the ExoMol database is being extended to systematically provide this additional data and other supplementary data in order to maximise the usefulness of the line lists [3]. A major new feature is the inclusion, albeit at a fairly crude level for most molecules, of pressurebroadening parameters and pressure-dependent absorption cross sections.

$\mathrm{H}_{2}{ }^{16} \mathrm{O}$ is one of the few molecules detected in the atmosphere of an exoplanet to date [4, 5]. In particular it has been identified in the atmospheres of several hot extrasolar giant planets including HD189733b [6, 7, ㅇ, 9, 10, 11], GJ 436b [12, 13], HD 209458b [14, 15], XO-1b [16, 15], HAT-P-1b [17], HD 179949b [18], WASP-19b [19], WASP-12b [20] and planets orbitting HR 8799 
[21, 22]. Test model calculations have shown that inclusion pressure broadening of the water spectrum significantly alters the observed spectrum of transiting hot Jupiter exoplanet [2].

Prior to the discovery of exoplanets, $\mathrm{H}_{2}{ }^{16} \mathrm{O}$ was already known to be a component of the atmospheres of cool stars and brown dwarfs [23, 24, 25, 26], where it can dominate the observed spectrum [27]. Recent discoveries have focused very cold brown dwarfs: Faherty et al. [28] made a tentative detection of water clouds in WISE J085510.83-071442.5 and Skemer et al. [29]

$\mathrm{H}_{2}{ }^{16} \mathrm{O}$ is one of the few molecules contained in the current release of HITEMP [30] which contains a comprehensive set of air- and self-broadening parameters. These were obtained using a diet based on physical principles and statistics [31] which had a strong bias toward near-room temperatures. As the bulk of the atmosphere of giant exoplanets and cool stars is composed of a $\mathrm{H}_{2}$ rich $\mathrm{H}_{2}$-He mix, molecular opacities in atmospheric models for these objects should incorporate pressure broadening due to $\mathrm{H}_{2}$ and He.

A number of works, reviewed in the next section, have provided pressure broadening parameters for broadening of $\mathrm{H}_{2}{ }^{16} \mathrm{O}$ by $\mathrm{H}_{2}$ and/or He for this purpose, although the spectral, temperature and pressure coverage is far from comprehensive. Altogether, detailed information for broadening of water vapour lines by $\mathrm{H}_{2}$ and $\mathrm{He}$ is available for around 1100 and 5000 lines respectively, while the most complete line list for water to date (BT2, [32]) contains around half a billion lines which is sufficientlly complete for temperatures up to $3000 \mathrm{~K}$. The temperature coverage, although appreciable for broadening by $\mathrm{H}_{2}(40-1500 \mathrm{~K})$, is more limited for broadened by $\mathrm{He}$ ( 83 $600 \mathrm{~K}$ ). Conversely the pressure coverage for broadening by He (up to $3 \mathrm{~atm}$ ) 
is more extensive than for broadening by $\mathrm{H}_{2}$ (up to $1330 \mathrm{mbar} \approx 1.3 \mathrm{~atm}$ ).

The first aim of this work is to provide $\mathrm{H}_{2}{ }^{16} \mathrm{O}-\mathrm{H}_{2}$ and $\mathrm{H}_{2}{ }^{16} \mathrm{O}-\mathrm{He}$ pressure broadening parameters suitable for temperatures up to $2000 \mathrm{~K}$ and pressures up to 10 bar. These parameters can be used to generate a pressure and temperature dependent Lorentzian half-width for every line in the BT2 line list, or indeed any water line list with at least rotational angular momentum $\left(J^{\prime}-J^{\prime \prime}\right)$ quantum assignments.

The implementation of full line lists in atmospheric modelling codes, although ideal, is often not practical due to their sheer size [33]. Hence the second aim of this work is to provide pressure-dependant absorption cross sections based on Voigt profiles for $\mathrm{H}_{2}{ }^{16} \mathrm{O}$ in a mixed $\mathrm{H}_{2} / \mathrm{He}(85 / 15 \%)$ environment for a range of temperatures $(\mathrm{T}=300-2000 \mathrm{~K})$ and pressures $(\mathrm{P}=$ 0.001 - 10 bar) relevant to exoplanet and cool star atmospheres.

\section{Previous work}

For the $\mathrm{H}_{2}{ }^{16} \mathrm{O}-\mathrm{H}_{2}$ system the most extensive experimental study was performed by Brown \& Plymate [34] who derived pressure broadened widths for 630 lines in the range $55-4045 \mathrm{~cm}^{-1}$ at room temperature. Other exclusively room temperature measurements have been carried out by Steyert et al. [35] (39 lines in the range $380-600 \mathrm{~cm}^{-1}$ ), Brown et al. [36] (4 lines around 1540 $\mathrm{cm}^{-1}$ ), Golubiatnikov [37] (1 line at $183 \mathrm{GHz}$ ), Lucchesinia, Gozzini \& Gabbanini [38] (15 lines in the range $820-830 \mathrm{~nm}$ ), and Zeninari et al. [39] (6 lines around $1.39 \mu \mathrm{m})$. The temperature and/or pressure dependence of the

collision induced widths for select $(2-12) \mathrm{H}_{2}{ }^{16} \mathrm{O}$ lines has been investigated by a handful studies [39, 40, 41, 42]. The works considering the widest range 
of temperatures and pressures are those by Langlois, Birbeck \& Hanson [41] and Zeninari et al. [39] respectively. Langlois, Birbeck \& Hanson combined two experimental set-ups, one incorporating a temperature controlled static cell (limited to $450 \mathrm{~K}$ ) the other a pressure driven shock tube, to determine the temperature dependence of the pressure broadened widths of $12 \mathrm{H}_{2}{ }^{16} \mathrm{O}$ lines over the range $300-1200 \mathrm{~K}$ with an expected accuracy of $\pm 25 \%$. The dependence could be roughly represented by a power law of decreasing collision induced line width with increasing temperature. Zeninari et al. made measurements of six $\mathrm{H}_{2}{ }^{16} \mathrm{O}$ lines at several pressures between 6 and 1330 mbar (ambient pressure $\approx 1000$ mbar) and concluded that the dependence could be described as a linear increase in collision induced line width with pressure.

Theoretically determined pressure broadened widths and their temperature dependence are available from Gamache, Lynch \& Brown [43], Faure et al. 44] and Drouin \& Wisenfeld [45]. Drouin \& Wisenfeld focused on three lines at cold temperatures (below $200 \mathrm{~K}$ ) while Gamache, Lynch \& Brown and Faure et al. aimed to provide information for hundreds of lines suitable for high temperature applications. The maximum temperature considered by Faure et al. was hotter (1500 K vs $750 \mathrm{~K}$ ) though Gamache, Lynch \& Brown produced parameters for more lines (386 vs 228). All three works focused on rotational transitions.

Several of the studies already mentioned [38, 35, 36, 37, 43, 40, 42, 39. also reported parameters for the $\mathrm{H}_{2}{ }^{16} \mathrm{O}-\mathrm{He}$ system. Although the most extensive studies of He-broadening coefficients of $\mathrm{H}_{2}{ }^{16} \mathrm{O}$ have been performed by Petrova and co-workers [46, 47, 48], and Solodov \& Starikov [49, 50]. Each of 
these works presented measurements of pressure broadened widths for tens of lines belonging to strong vibrational bands including $\nu_{2}+\nu_{3}$ [49, 50, 47, 48], $\nu_{1}+\nu_{2}$ [50, 47, 48], $\nu_{1}+\nu_{2}+\nu_{3}$ [46, 47] and $2 \nu_{3}$ and $\nu_{1}+2 \nu_{1}$ [48] at room temperature and varying pressures. All found the pressure dependence to be linear (up to 3 atm [47, 48]). Petrova et al. [47] combined their own measurements with literature data to determine the vibrationally and rotationally dependent intermolecular potential for the $\mathrm{H}_{2}{ }^{16} \mathrm{O}$-He system. This potential was used by Petrova et al. [47, 48] to compute, and fit an analytical expression to, helium pressure broadened width for transitions belonging to 11 and 13 vibrational bands respectively with rotational quantum numbers $J$ and $K_{a}$ up to 14 in the temperature interval $83 \leq T \leq 600 \mathrm{~K}$. The temperature dependence in the model was refined by comparison to previous temperature dependent studies Goyette \& De Lucia [51] (1 line at $183 \mathrm{GHz}, 80$ - $600 \mathrm{~K}$ ) and Godon \& Bauer [52] (2 lines at 183 and $380 \mathrm{GHz}, 300$ - $390 \mathrm{~K}$ ). In addition room temperature pressure broadened widths for $\mathrm{H}_{2}{ }^{16} \mathrm{O}-\mathrm{He}$ have been measured by Lazarev et al. [53] (1 line at $14397.4 \mathrm{~cm}^{-1}$ ), Poddar et al. [54] (14 lines in the range 11,988-12,218 $\mathrm{cm}^{-1}$ ), Claveau et al. [55] (14 lines in the range $1850-2140 \mathrm{~cm}^{-1}$ ) and Claveau \& Valentin [56] (10 lines in the range $\left.1170-1440 \mathrm{~cm}^{-1}\right)$. Claveau et al. and Claveau \& Valentin also investigated narrowing due to dynamic confinement (Dicke narrowing [57, 58]). 


\section{Calculation of $\mathrm{H}_{2}$ and He pressure induced line widths of $\mathrm{H}_{2}{ }^{16} \mathrm{O}$ spectral lines}

\subsection{Theoretical techniques used}

Two calculation techniques are used to determine the $\mathrm{H}_{2}{ }^{16} \mathrm{O}-\mathrm{H}_{2}$ and $\mathrm{H}_{2}{ }^{16} \mathrm{O}$-He Lorentzian half-widths. These techniques distinguish between rigorous quantum numbers, namely total angular momentum $J$ and total sym-

metry $\left(\Gamma_{\text {tot }}\right)$, which corresponds to parity and ortho/para designation, and approximate projections of the rotational motion $\left(K_{a}, K_{c}\right)$. The techniques are:

1. the averaged energy difference (AED) method [59] is used in cases where the complete set of quantum numbers (both rigorous and approximate) of a transition are known;

2. the $J^{\prime} J^{\prime \prime}$ - dependence technique [60] has been applied in cases where only rigorous (total angular momentum $J$ and total symmetry) were known.

These approaches are described in detail in the respective papers, thus only the main features are outlined below.

The averaged energy difference method allows the calculation of line widths of asymmetric top molecules with approximately the same precision as in modern theoretical and experimental methods without the need for a complicated calculation scheme. The approach is based on matching the so called coupled energy state difference with a line broadening value. Based on the modified Robert-Bonamy formalism, the expression for the half-widths $\gamma_{i f}$ is given by [61, 62]: 


$$
\gamma_{i f}=\frac{n_{b}}{2 \pi c} \int_{0}^{+\infty} v f(v) d v \int_{0}^{+\infty} 2 \pi b d b\left[1-\cos \left(S_{1}+\operatorname{Im}\left(S_{2}\right)\right) e^{-\operatorname{Re}\left(S_{2}\right)}\right]
$$

where indices $i$ and $f$ specify initial and final energy states, $n_{b}$ is the number density of perturbers, $b$ is the impact parameter and $v$ is the relative initial velocity and $f(v)$ is the MaxwellBoltzmann distribution function [59, 61, 62]. Real $S_{1}$ and complex $S_{2}=\operatorname{Re}\left(S_{2}\right)+i \operatorname{Im}\left(S_{2}\right)$ are the first and second order terms in the expression for the scattering matrix. These terms depend on collision dynamics, the intermolecular potential and on the ro-vibrational states of the molecule (and collision induced transitions between these states). Expressions for $S_{1}$ and $S_{2}$ can in found in Refs. [59, 63, 64, 65].

The aim is to estimate how values of $\operatorname{Re}\left(\mathrm{S}_{2}\right)$ vary with different lines of interest. Coupled energy state differences $\tilde{\omega}_{i f}$ between an $\mathrm{H}_{2}{ }^{16} \mathrm{O}$ line of interest and other coupled lines ( $\tilde{\omega}_{i f}$ is defined explicitly in [59]) are derived for thousands of lines. The averaged energy differences for lines with experimentally-determined collision-induced widths are then used to match averaged state energy differences to line broadening values using a fitting formula.

The dependence of broadening parameters on the averaged energy difference of the coupled states is smooth. Figure 11 illustrates this for the $\mathrm{H}_{2}{ }^{16} \mathrm{O}-\mathrm{H}_{2}$ line widths. This figure demonstrates that one can estimate corresponding widths with a simple fitting formula. For other systems such $\mathrm{N}_{2}$ broadening of water the observed data has been found to lie almost exactly on a smooth curve [66]; here the scatter reflects, at least in part, uncertainty in the experimental data. The reconstructed widths obtained from the fitting formula agree well with the experimental values as shown in Figure 2 for 


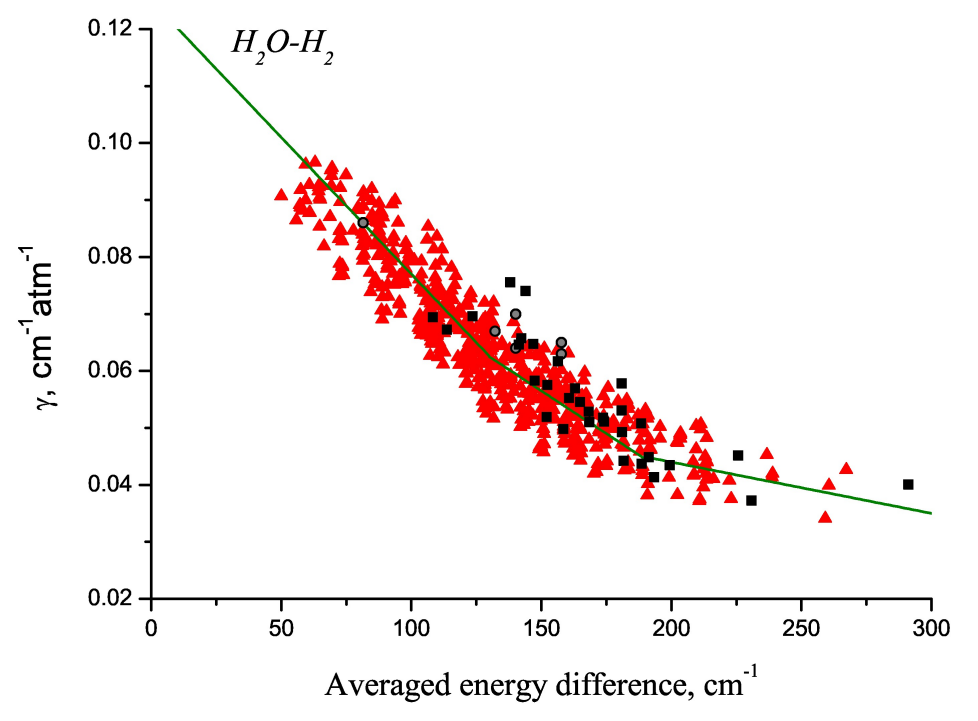

Figure 1: Dependence of $\mathrm{H}_{2}{ }^{16} \mathrm{O}-\mathrm{H}_{2}$ broadening coefficients on averaged coupled energy state difference at $296 \mathrm{~K}$; experimental data: rotational band and fundamentals [34, red triangles; rotational band [35, black squares; $v_{1}+v_{3}$ and $2 v_{2}$ [41], gray cirles.

$\mathrm{H}_{2}{ }^{16} \mathrm{O}-\mathrm{H}_{2}$. This method requires assignment to normal modes (via quantum numbers $v_{1}, v_{2}, v_{3}, K_{a}$ and $K_{c}$ ). However, these quantum numbers are not always known or, indeed, always valid [67].

For cases where only good quantum numbers $\left(J\right.$ and $\left.\Gamma_{\text {tot }}\right)$ are defined, the $J^{\prime}-J^{\prime \prime}$ dependence technique can be applied. Available widths are compiled and averaged for each value of $J^{\prime}$ per branch (P, Q and R) and total symmetry (ortho/para). The resulting values are used to derive functions which describe the dependence of the averaged width on $J^{\prime}$ per branch and per symmetry (if there is appreciable difference for the latter two). This method applied to $\mathrm{H}_{2}{ }^{16} \mathrm{O}-\mathrm{H}_{2}$ and $\mathrm{H}_{2}{ }^{16} \mathrm{O}-\mathrm{He}$ is illustrated in Figure 3 . 


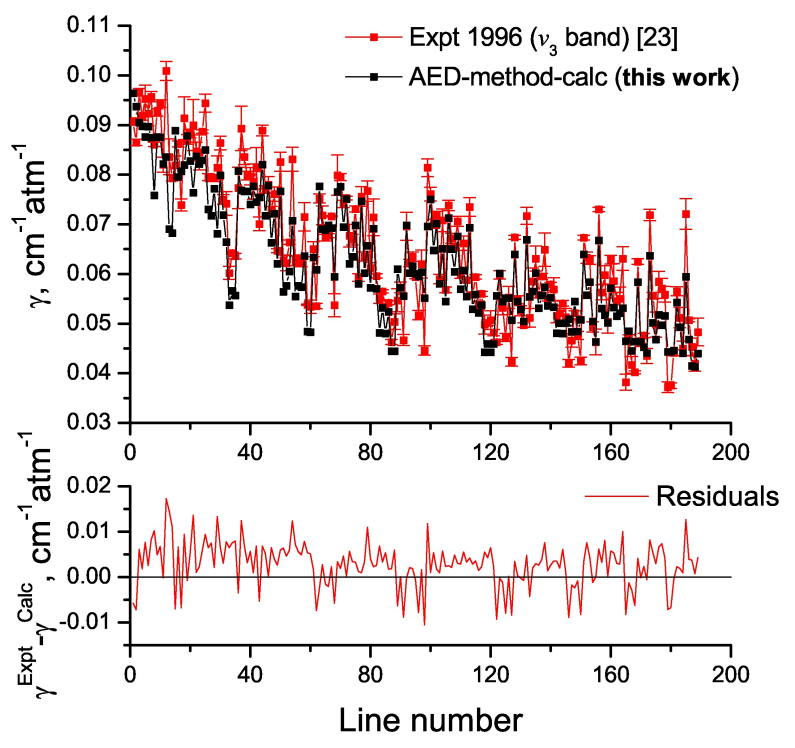

Figure 2: Comparison of reconstructed $\mathrm{H}_{2}{ }^{16} \mathrm{O}-\mathrm{H}_{2}$ pressure dependent line widths with experimental values for the $v_{3}$ band [34] at $296 \mathrm{~K}$.

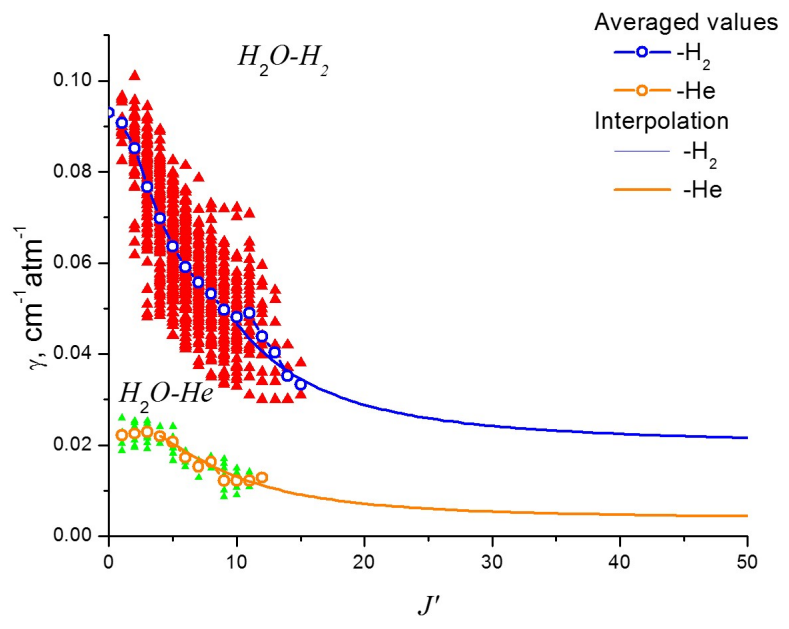

Figure 3: Dependence of $\mathrm{H}_{2}{ }^{16} \mathrm{O}-\mathrm{H}_{2}$ and $\mathrm{H}_{2}{ }^{16} \mathrm{O}-\mathrm{He}$ broadening coefficients on $J^{\prime}$ at $296 \mathrm{~K}$. 


\subsection{The $\mathrm{H}_{2}{ }^{16} \mathrm{O}-\mathrm{H}_{2}$ and $\mathrm{H}_{2}{ }^{16} \mathrm{O}$-He line widths}

Both approaches described in the previous section were used to obtain separate $\mathrm{H}_{2}{ }^{16} \mathrm{O}-\mathrm{H}_{2}$ and $\mathrm{H}_{2}{ }^{16} \mathrm{O}-\mathrm{He}$ Lorentzian half-widths for BT2 lines in the range $500-10,000 \mathrm{~cm}^{-1}$ with an intensity that exceeds $1 \times 10^{-30} \mathrm{~cm} /$ molecule in the temperature range 300 - $2000 \mathrm{~K}$. Vibrational dependence is neglected as it is found to change the value of the half-widths by only a few percent. Dependence on $K_{c}$ and total symmetry was found to be much smaller than dependence on $K_{a}$ and $J^{\prime}-J^{\prime \prime}$ respectively.

Our calculated values range between $0.0208-0.0927 \mathrm{~cm}^{-1} \mathrm{~atm}^{-1}$ for broadening by $\mathrm{H}_{2}$ and $0.0043-0.0229 \mathrm{~cm}^{-1}$ atm $^{-1}$ for broadening by He at $296 \mathrm{~K}$. The average ratio between the $\mathrm{H}_{2}$ to He pressure induced line widths is 4.2. Broadening of water by $\mathrm{H}_{2}$ is much stronger than by He because the $\mathrm{H}_{2}$ molecule has a quadrupole moment. The electrostatic potential is given by an expansion of the charge distribution in terms of the electric moments of the molecules. So, the main contribution into $\mathrm{H}_{2} \mathrm{O}-\mathrm{H}_{2}$ broadening value is from the dipole-quadrupole interaction, besides this term there is also a quadrupole-quadrupole interaction contribution. Helium does not have any electric moments, so only the polarization potential (interactions between induced moments) gives the contribution, which is very much weaker.

Tables 1 and 2 present a summary of the comparison of our calculated values with available experimental and previous theoretical studies. The root mean square deviations (RMSD) between this work and other works is given as a percentage of the current values. For the $\mathrm{H}_{2}{ }^{16} \mathrm{O}-\mathrm{H}_{2}$ system the RMSD is within $20 \%$ or the uncertainty quoted in the given reference for all studies. It should be noted that for the comparison to Zeninari et al. the RMSD 
Table 1: Root mean square deviations (RMSD) of calculated values from available data on $\mathrm{H}_{2}{ }^{16} \mathrm{O}-\mathrm{H}_{2}$ broadening; $\gamma$ is the half-width and $n$ the temperature exponent. In many cases there are no experimental data available for comparison.

\begin{tabular}{lrrr}
\hline Reference & Number of Transitions & RMSD $\gamma$ & RMSD $n$ \\
\hline Experiment & 39 & $10.4 \%$ & - \\
Steyert et al. [35] & 630 & $7.4 \%$ & - \\
Brown \& Plymate et al. [34] & 4 & $8.8 \%$ & - \\
Brown et al. [36] & 1 & $13.9 \%$ & - \\
Golubiatnikov [37] & 6 & $19.1 \%$ & - \\
Zeninari et al. [39] & 11 & $17 \%$ & $36.9 \%$ \\
Langlois, Birbeck \& Hanson [41] & 2 & $7.3 \%$ & - \\
Dutta et al. [42] & & & \\
Theory & 386 & $6.2 \%$ & \\
Gamache, Lynch and Brown [43] & 32 & & $4.9 \%$ \\
Faure et al. 44$]$ & 228 & $24.1 \%$ & $18.2 \%$ \\
\hline
\end{tabular}

of $19.1 \%$ is mainly down to the $6_{60} \leftarrow 6_{61}$ transition which is in fact an unresolved doublet [39]. The RMSD for the remaining five transitions is 8.4 $\%$.

For the $\mathrm{H}_{2}{ }^{16} \mathrm{O}-\mathrm{He}$ system the RMSD is within $26 \%$ for all studies. Again for the comparison to Zeninari et al. the RMSD of $15.0 \%$ is mainly down to the $6_{60} \leftarrow 6_{61}$ transition. The RMSD for the remaining five transitions is $8.6 \%$. 
Table 2: Root mean square deviations (RMSD) of calculated values from available data on $\mathrm{H}_{2}{ }^{16} \mathrm{O}$-He broadening; $\gamma$ is the half-width and $n$ the temperature exponent.

\begin{tabular}{|c|c|c|c|}
\hline Reference & Number of Transitions & $\operatorname{RMSD} \gamma$ & $\operatorname{RMSD} n$ \\
\hline \multicolumn{4}{|l|}{ Experiment } \\
\hline Petrova et al. 48 & 103 & $17.1 \%$ & - \\
\hline Petrova et al. [47] & 150 & $17.7 \%$ & - \\
\hline Petrova et al. 46] & 105 & $15.5 \%$ & - \\
\hline Solodov \& Starikov [49] & 32 & $14.0 \%$ & - \\
\hline Solodov \& Starikov [50] & 53 & $13.9 \%$ & - \\
\hline Goyette \& De Lucia 51 & 1 & $1.7 \%$ & $42.2 \%$ \\
\hline Godon \& Bauer 52 ] & 2 & $10.3 \%$ & $13.6 \%$ \\
\hline Poddar et al. [54] & 14 & $14.0 \%$ & - \\
\hline Claveau \& Valentin [56] & 10 & $25.7 \%$ & - \\
\hline Claveau et al. 55 & 14 & $24.7 \%$ & - \\
\hline Steyert et al. 35] & 39 & $23.3 \%$ & - \\
\hline Brown et al. 36 & 4 & $11.1 \%$ & - \\
\hline Dutta et al. 42 & 2 & $9.2 \%$ & - \\
\hline Zeninari et al. 39 . & 6 & $15.0 \%$ & - \\
\hline Golubiatnikov 37. & 1 & $4.0 \%$ & - \\
\hline Lazarev et al. 53 ] & 1 & $7.1 \%$ & - \\
\hline \multicolumn{4}{|l|}{ Theory } \\
\hline \multirow[t]{2}{*}{ Gamache, Lynch and Brown 43} & 386 & $6.2 \%$ & \\
\hline & 32 & & $4.9 \%$ \\
\hline
\end{tabular}




\subsection{Temperature and pressure dependence of the $\mathrm{H}_{2}{ }^{16} \mathrm{O}-\mathrm{H}_{2} / \mathrm{He}$ line widths}

In order to determine the temperature dependence, the half-width calculations were made at the following temperatures: $T=300,400,500,600$, 700, 800, 900, 1000, 1200, 1400, 1600, 1800, $2000 \mathrm{~K}$. The temperature dependence, represented by exponent $n$, was obtained by fitting to the standard relation:

$$
\gamma(T)=\gamma_{\mathrm{ref}} \times\left(\frac{T_{\mathrm{ref}}}{T}\right)^{n} .
$$

where $T_{\text {ref }}=296 \mathrm{~K}$ is the reference temperature, $\gamma_{\text {ref }}$ is the Lorentzian halfwidth at reference temperature and $n$ is the temperature exponent. The temperature exponents vary from 0.866 to 0.027 for broadening by $\mathrm{H}_{2}$ and from 0.5 to 0.02 for broadening by He. Comparisons with experimentally derived and previously calculated values are summarised in Tables 1 and 2 .

Recently Wilzewski et al. 68] commented that a single power law of the form Eq. (2) only works well within relatively narrow temperature intervals. However they also note that there is not enough experimental data to characterise an alternative model at present, and we find Eq. (2) reproduces our calculated values for the temperature range 300 - $2000 \mathrm{~K}$ to sufficient accuracy (within 3\%). For high accuracy treatments other issues arise with use of the Voigt profile [69].

The pressure dependence of the Lorentzian half-widths in the range 0.001 - 10 bar is assumed to be linear:

$$
\gamma(P)=\gamma_{\text {ref }} \times\left(\frac{P}{P_{\text {ref }}}\right) .
$$

where $P_{\text {ref }}=1$ bar is the reference pressure. This is indicated by available pressure dependent experimental investigations up to around ambient pressure for broadening by $\mathrm{H}_{2}[39$, and up to around 3 atmospheres ( $\approx 3$ bar) 
for broadening by $\mathrm{He}$ [47, 48]. Although measurements at the high end of the pressure range ( $\sim 10$ bar) would be helpful to verify this; in particular high pressure leads to three-body effects which are implicitly neglected in our formulation.

\subsection{The .broad files}

In principle parameters were calculated for around 4 million BT2 lines with an intensity that exceeds $1 \times 10^{-30} \mathrm{~cm} /$ molecule in the temperature range 300 - $2000 \mathrm{~K}$. In practice, since vibrational dependence was neglected and dependence on total symmetry and rotational quantum number $K_{c}$ was found to be comparatively small, the results comprise of around 23,000 widths that depend on $J^{\prime}, J^{\prime \prime}, K_{a}{ }^{\prime}$ and $K_{a}{ }^{\prime \prime}$, and 100 widths that depend on $J^{\prime}$ and $J^{\prime \prime}$ only. This can be represented in new ExoMol format [3] as two .broad files:

1H2-160__H2__NLAD. broad, extract shown in Table 3 1H2-160__He__NLAD. broad, extract shown in Table 4

These files and Eq.(4) below can be used to generate a pressure and temperature dependent Lorentzian half-width for any water vapour line, with at least $J^{\prime}-J^{\prime \prime}$ quantum assignments, for a pure $\mathrm{H}_{2}$, pure $\mathrm{He}$ or mixed $\mathrm{H}_{2} / \mathrm{He}$ environment.

$$
\gamma(T, P)=\left(\frac{T_{\text {ref }}}{T}\right)^{n} \times\left(\frac{P}{P_{\text {ref }}}\right) \times \sum_{b} \gamma_{\text {ref }, \mathrm{b}} p_{b} .
$$

where $\gamma_{\text {ref,b }}$ is the Lorentzian half-width due to a specific broadener in units $\mathrm{cm}^{-1} /$ bar (ExoMol convention) and $p_{b}$ is the partial pressure of the broad-

ener. Note that to convert from $\mathrm{cm}^{-1} /$ bar used by ExoMol to $\mathrm{cm}^{-1} / \mathrm{atm}$ 
used by Hitran requires $\gamma$ to be mutiplied by 1.01325 .

The . broad files presented as part of this work differ slightly from those included in the ExoMol database. The . broad files provided with the ExoMol line lists, described in [3], contain data from multiple sources while the . broad files available as supplementary data to this work contain data from only one source, the current work. Hence, the file names are appended with a dataset name NLAD. We recomend the use of the full . broad file presented on the ExoMol website as this includes both line-by-line parameters where available, which are essential for detailed and high resolution studies, while the current model which allows a width to be generated for every molecular line.

\section{Computation of $\mathrm{H}_{2}{ }^{16} \mathrm{O}$ absorption cross-sections}

\subsection{Method}

The high resolution cross section is calculated on an evenly spaced wavenumber grid, $\tilde{\nu}_{i}$, defining bins of width $\Delta \tilde{\nu}$. A Voigt profile is used to model the joint contributions from thermal and collision induced broadening:

$$
f_{V}\left(\tilde{\nu}, \tilde{\nu}_{0 ; j}, \alpha_{j}, \gamma_{j}\right)=\frac{1}{\sqrt{\pi}} \frac{\sqrt{\ln 2}}{\alpha_{j}} \operatorname{wofz}\left(\frac{\tilde{\nu}-\tilde{\nu}_{0 ; j}}{\alpha_{j}} \sqrt{\ln 2}+i \frac{\gamma_{j}}{\alpha_{j}} \sqrt{\ln 2}\right)
$$

where wofz is the scaled complex complementary error function, also known as the Faddeeva function. This is calculated using the Faddeeva package [70]. $\tilde{\nu}_{0 ; j}$ is the line centre, $\gamma_{j}$ is the Lorentzian half-width at half-maximum and $\alpha_{j}$ is the Doppler half-width at half-maximum given by: 
Table 3: 1H2-160_H2__NLAD.broad: Extract of $\mathrm{H}_{2}{ }^{16} \mathrm{O}-\mathrm{H}_{2}$ broad file: portion of the file (upper part); field specification (lower part).

\begin{tabular}{lllllll}
\hline a3 & 0.0690 & 0.502 & 5 & 4 & 1 & 3 \\
a3 & 0.0580 & 0.424 & 6 & 6 & 3 & 3 \\
a3 & 0.0754 & 0.541 & 3 & 3 & 0 & 1 \\
$\ldots$ & & & & & & \\
a1 & 0.0342 & 0.253 & 14 & 15 & & \\
a1 & 0.0328 & 0.241 & 15 & 16 & & \\
a1 & 0.0317 & 0.240 & 16 & 17 & &
\end{tabular}

\begin{tabular}{llll}
\hline Field & Fortran Format & C format & Description \\
\hline code & A2 & $\% 2 \mathrm{~s}$ & Code identifying quantum number set following $J^{\prime \prime *}$ \\
$\gamma_{\text {ref }}$ & F6.4 & $\% 6.4 \mathrm{f}$ & Lorentzian half-width at reference temperature and pressure $\mathrm{cm}^{-1} / \mathrm{bar}$ \\
$n$ & $\mathrm{~F} 5.3$ & $\% 5.3 \mathrm{f}$ & Temperature exponent \\
$J^{\prime \prime}$ & $\mathrm{I} 7$ & $\% 7 \mathrm{~d}$ & Lower $J$-quantum number \\
$J^{\prime}$ & $\mathrm{I} 7$ & $\% 7 \mathrm{~d}$ & Upper $J$-quantum number \\
$K_{a}^{\prime \prime}$ & $\mathrm{I} 2$ & $\% 2 \mathrm{~d}$ & Lower rotational quantum number \\
$K_{a}{ }^{\prime}$ & $\mathrm{I} 2$ & $\% 2 \mathrm{~d}$ & Upper rotational quantum number \\
\hline
\end{tabular}

*Code definitions: a3 $=$ parameters presented as a function of $J^{\prime \prime}$ (compulsory) and $J^{\prime}$, $K_{a}{ }^{\prime \prime}$ and $K_{a}{ }^{\prime}$. a1 $=$ parameters presented as a function of $J^{\prime \prime}$ (compulsory) and $J^{\prime}$. 
Table 4: $1 \mathrm{H} 2-160 \_$He__NLAD.broad: Extract of $\mathrm{H}_{2}{ }^{16} \mathrm{O}-\mathrm{He}$ broad file: portion of the file (upper part); field specification (lower part).

\begin{tabular}{lllllll}
\hline a3 & 0.0213 & 0.269 & 5 & 4 & 1 & 3 \\
a3 & 0.0183 & 0.241 & 6 & 6 & 3 & 3 \\
a3 & 0.0226 & 0.320 & 3 & 3 & 0 & 1 \\
$\ldots$ & & & & & & \\
a1 & 0.0092 & 0.179 & 14 & 15 & & \\
a1 & 0.0086 & 0.145 & 15 & 16 & & \\
a1 & 0.0082 & 0.148 & 16 & 17 & &
\end{tabular}

\begin{tabular}{llll}
\hline Field & Fortran Format & C format & Description \\
\hline code & A2 & $\% 2 \mathrm{~s}$ & Code identifying quantum number set following $J^{\prime \prime *}$ \\
$\gamma_{\text {ref }}$ & F6.4 & $\% 6.4 \mathrm{f}$ & Lorentzian half-width at reference temperature and pressure in $\mathrm{cm}^{-1} / \mathrm{bar}$ \\
$n$ & F5.3 & $\% 5.3 \mathrm{f}$ & Temperature exponent \\
$J^{\prime \prime}$ & I7 & $\% 7 \mathrm{~d}$ & Lower $J$-quantum number \\
$J^{\prime}$ & I7 & $\% 7 \mathrm{~d}$ & Upper $J$-quantum number \\
$K_{a}^{\prime \prime}$ & I2 & $\% 2 \mathrm{~d}$ & Lower rotational quantum number \\
$K_{a}{ }^{\prime}$ & I2 & $\% 2 \mathrm{~d}$ & Upper rotational quantum number \\
\hline
\end{tabular}

*Code definitions: a $3=$ parameters presented as a function of $J^{\prime \prime}$ (compulsory) and $J^{\prime}$, $K_{a}{ }^{\prime \prime}$ and $K_{a}{ }^{\prime}$. a1 $=$ parameters presented as a function of $J^{\prime \prime}$ (compulsory) and $J^{\prime}$. 


$$
\alpha=\sqrt{\frac{2 k T \ln 2}{m}} \frac{\tilde{\nu}_{0 ; j}}{c}
$$

at temperature $T$ in $\mathrm{K}$ for a molecule of mass $m$ in $\mathrm{kg}$. Note that in the limit $\alpha \gg \gamma$, the profile reduces to a Gaussian:

$$
f_{G}\left(\tilde{\nu}, \tilde{\nu}_{0 ; j}, \alpha_{j}\right)=\sqrt{\frac{\ln 2}{\pi}} \frac{1}{\alpha_{j}} \exp \left(-\frac{\left(\tilde{\nu}-\tilde{\nu}_{0 ; j}\right)^{2} \ln 2}{\alpha_{j}^{2}}\right)
$$

whereas for $\alpha \ll \gamma$ the profile reduces to a Lorentzian:

$$
f_{L}\left(\tilde{\nu}, \tilde{\nu}_{0 ; j}, \gamma_{j}\right)=\frac{\gamma_{j}}{\pi} \frac{1}{\left(\tilde{\nu}-\tilde{\nu}_{0 ; j}\right)^{2}+\gamma_{j}^{2}}
$$

However a Voigt profile is evaluated for every absorption line in all calculations of cross sections presented in this work. The cross section for each bin is the sum of the contributions from individual lines:

$$
\sigma_{i}=\sum_{j} \sigma_{i j}
$$

where:

$$
\sigma_{i j}=S_{j} f_{V}\left(\tilde{\nu}, \tilde{\nu}_{0 ; j}, \alpha_{j}, \gamma_{j}\right)
$$

$S_{j}$ is the line intensity in units of $\mathrm{cm}$ per molecule given by:

$$
S_{j}=\frac{A_{j}}{8 \pi c} \frac{g_{j}^{\prime} \exp \left(-c_{2} E_{j}^{\prime \prime} / T\right)}{\tilde{\nu}_{0 ; j}^{2} Q(T)}\left(1-\exp \left(-\frac{c_{2} \tilde{\nu}_{0 ; j}}{T}\right)\right)
$$

Here, $g_{j}^{\prime}$ and $E_{j}^{\prime \prime}$ are the total upper-state degeneracy and lower-state energy respectively, $A_{j}$ is the Einstein A coefficient for the transition and $c_{2}=h c / k_{B}$ is the second radiation constant. $\tilde{\nu}_{0 ; j}$ and $A_{j}$ were taken from the BT2 line 
list while the molecular partition function, $\mathrm{Q}(\mathrm{T})$, was obtained from the tabulated values of Vidler \& Tennyson [71].

When evaluating a Voigt profile it is necessary to select an appropriate profile grid resolution and evaluation width in order to adequately sample the contribution from the profile whilst considering the computational cost. For the profile grid resolution we adopted the staggered wavenumber grid spacings of [33] (see Table 5). These spacings are well below the Voigt width for the pressure and temperature range considered, which is essential for Eq. (10) to be valid.

For the profile evaluation width we adopted a cut-off of $200 \times\left(\alpha_{j}+\gamma_{j}\right)$ either side of the centroid. We find that this is sufficient to capture the contributions from the wings in a manner that adapts with both pressure and temperature.

We note Hedges \& Madhusudhan [72] recently investigated the effect of various factors, including the profile grid resolution and evaluation width, on pressure dependent absorption cross sections for application to exoplanet atmospheres. Hedges \& Madhusudhan proposed a more computationallyefficient grid resolution, a pressure adaptive grid, than the staggered grid employed by this work. However, they comment that the pressure adaptive grid resulted in a small loss of opacity $(\sim 2 \%)$ compared to the staggered grid at the high end of the pressure range $(\mathrm{P} \sim 10 \mathrm{bar})$. Hence we opted for the staggered grid. For the profile evaluation width Hedges \& Madhusudhan compared two methods, a pressure adaptive fixed cut-off and a cut-off evaluated per line as a multiple of Lorentzian half-widths, to their approach, a cut-off evaluated per line as a multiple of effective Voigt half-widths. Their 
Table 5: Summary of the grid spacings for the cross sections calculated in difference wavenumber regions

\begin{tabular}{lc}
\hline Wavenumber range $\mathrm{cm}^{-1}$ & Grid spacing $\mathrm{cm}^{-1}$ \\
\hline $10-100$ & $10^{-5}$ \\
$100-1000$ & $10^{-4}$ \\
$1000-10,000$ & $10^{-3}$ \\
$10,000-30,000$ & $10^{-2}$ \\
\hline
\end{tabular}

effective Voigt half-width was defined as [72]:

$$
\gamma_{V} \approx 0.5346 \gamma_{L} \sqrt{0.2166 \gamma_{L}^{2}+\gamma_{G}^{2}}
$$

where $\gamma_{G}=\alpha_{j}, \gamma_{L}=\gamma_{j}$ and $\gamma_{V}<\left(\alpha_{j}+\gamma_{j}\right)$ for comparison to this work. Hedges \& Madhusudhan found that both the fixed cut-off method and Lorentzian half-width analysis resulted in significant ( $>40 \%$ ) opacity loss at the low end of the pressure range $(\mathrm{P}<0.01$ bar $)$ compared to the Voigt half-width analysis. As our approach is very similar to Hedges \& Madhusudhan we should also avoid this loss of opacity.

\subsection{Results}

Pressure dependent absorption cross sections for $\mathrm{H}_{2}{ }^{16} \mathrm{O}$ in a mixed $\mathrm{H}_{2} / \mathrm{He}$ environment ( $85 / 15 \%$ by number) are calculated on a fixed temperature and pressure grid (see Table 6) using the BT2 line list, pressure broadening parameters determined as part of this work (Section 1) and the method described above. However, no attempt is made include contributions to the opacity from the water vapour continuum or water dimer absorption. 
Table 6: Temperatures and Pressures at which $\mathrm{H}_{2}{ }^{16} \mathrm{O}$ cross sections are calculated

\begin{tabular}{cccc}
\hline Temperature $(\mathrm{K})$ & & & \\
\hline 300 & 400 & 500 & 600 \\
700 & 800 & 900 & 1000 \\
1200 & 1400 & 1600 & 1800 \\
2000 & & & \\
\hline Pressure (bar) & & & \\
\hline 0.001 & 0.003 & 0.005 & 0.01 \\
0.02 & 0.04 & 0.08 & 0.1 \\
0.3 & 0.6 & 0.9 & 1.0 \\
1.2 & 1.5 & 2.0 & 2.5 \\
3.0 & 4.0 & 5.0 & 6.0 \\
8.0 & 10.0 & & \\
\hline
\end{tabular}

The cross sections were calculated between 10 and $30,000 \mathrm{~cm}^{-1}$ using the staggered wavenumber grid given in Table 5 and then binned to a common grid spacing of $0.01 \mathrm{~cm}^{-1}$. Each region was calculated to overlap with its neighbours by at least $1 \mathrm{~cm}^{-1}$, which we find to be sufficient to avoid discontinuities when they are binned to a common grid spacing.

Calculated cross sections for a range of temperatures at a single pressure and a range of pressures at a single temperature are shown in Figures 4 and 5. A change in temperature predominately influences the shape of the cross section (Figure 4) while a change in pressure results in a redistribution of opacity (Figure 5). 


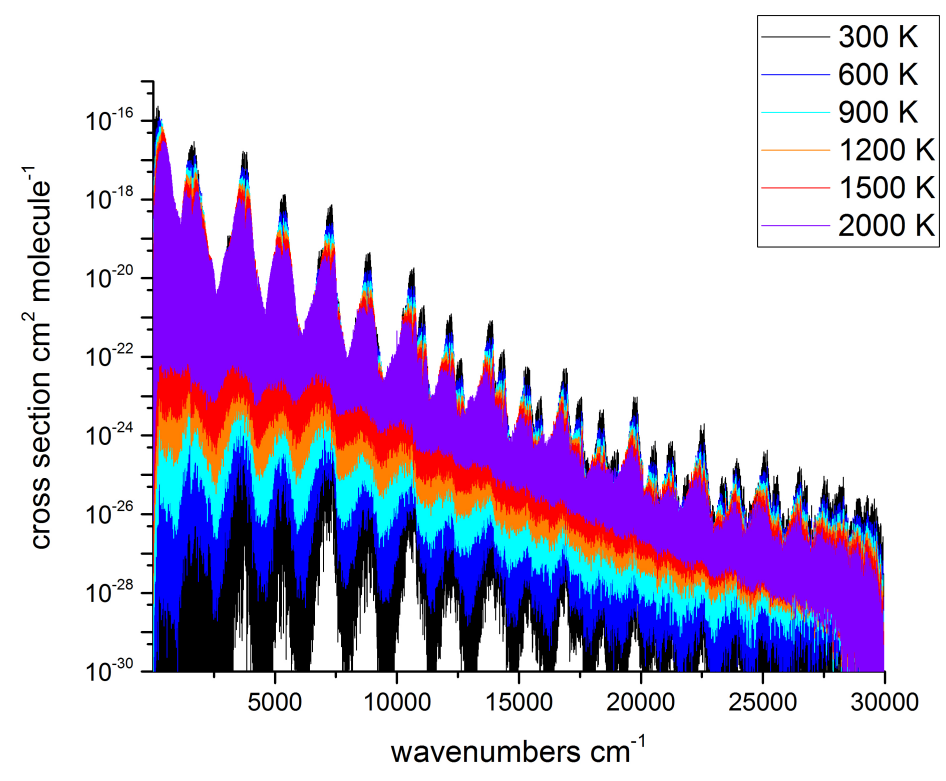

Figure 4: $\mathrm{H}_{2}{ }^{16} \mathrm{O}$ cross sections in a mixed $\mathrm{H}_{2}-\mathrm{He}(85 / 15 \%)$ environment calculated at 0.01 bar and temperatures in the range $300-2000 \mathrm{~K}$. A cut-off of $200 \times\left(\alpha_{j}+\gamma_{j}\right)$ was used (see text). 


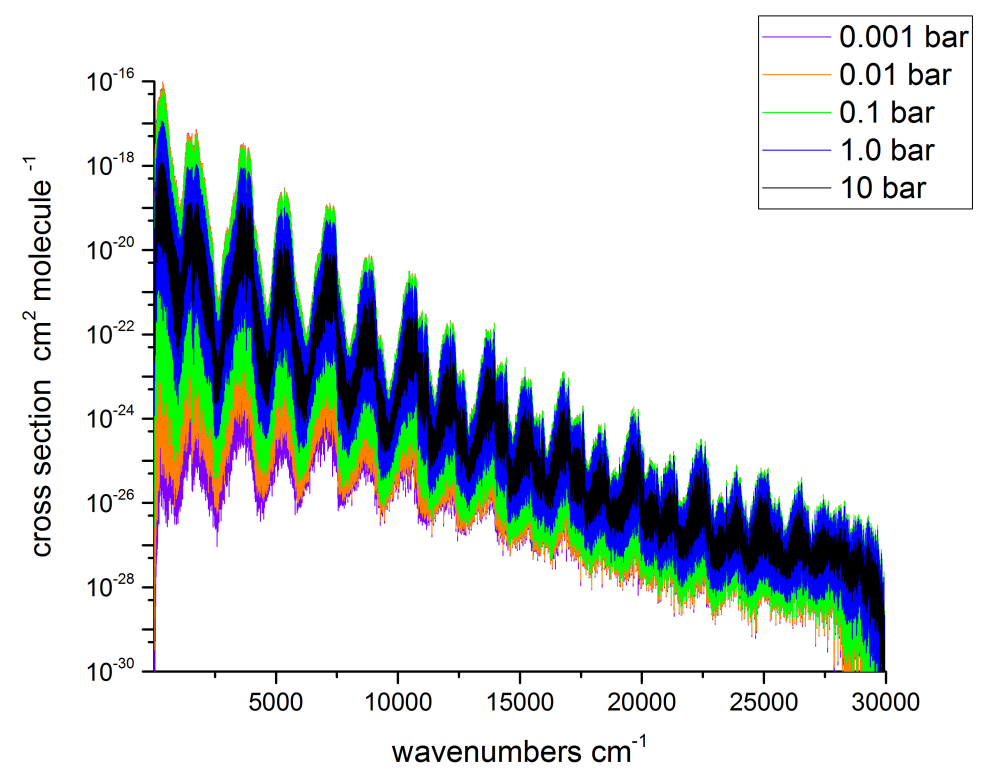

Figure 5: $\mathrm{H}_{2}{ }^{16} \mathrm{O}$ cross sections in a mixed $\mathrm{H}_{2}-\mathrm{He}(85 / 15 \%)$ environment calculated at 1000 $\mathrm{K}$ and pressures in the range $0.001-10$ bar. A cut-off of $200 \times\left(\alpha_{j}+\gamma_{j}\right)$ was used (see text). 


\subsection{Interpolation of cross-sections between temperatures and pressures}

Cross sections are provided for 12 temperatures and 22 pressures between $300-2000 \mathrm{~K}$ and 0.001 and 10 bar respectively (see Table 6). A cross section at intermediate conditions may be obtained by first interpolating on the temperature grid using either Eq. (14) or (16) below [33], then interpolating on the pressure grid using Eq. (19) below. The interpolation residual is expressed as a percentage of the corresponding absorption cross-section:

$$
\delta \sigma_{\max }^{\%}=\max \left(\frac{\left|\sigma_{i, \text { calc }}-\sigma_{i, \text { interp }}\right|}{\sigma_{i, \text { calc }}}\right) \times 100
$$

To obtain cross sections at an intermediate temperature $T$, interpolation between cross sections computed for a higher temperature $T_{2}$ and a lower temperature $T_{1}$ may be performed linearly [33]:

$$
\sigma_{i}=\sigma_{i}\left(T_{1}\right)+m\left(T-T_{1}\right)
$$

where:

$$
m=\frac{\sigma_{i}\left(T_{2}\right)-\sigma_{i}\left(T_{1}\right)}{T_{2}-T_{1}}
$$

Or using a more accurate exponential model [33]:

$$
\sigma_{i}=a_{i} e^{\frac{b_{i}}{T}}
$$

where:

$$
b_{i}=\left(\frac{1}{T_{2}}-\frac{1}{T_{1}}\right)^{-1} \ln \frac{\sigma_{i}\left(T_{1}\right)}{\sigma_{i}\left(T_{2}\right)}
$$

and: 


$$
a_{i}=\sigma_{i}\left(T_{1}\right) e^{\frac{b_{i}}{T}}
$$

The error in the cross sections introduced by the more accurate interpolation scheme does not exceed $1.64 \%$ which is less than the estimated uncertainty in the ab initio line lists.

To obtain cross sections at an intermediate pressure $P$, interpolation between cross sections computed for a higher pressure $P_{2}$ and a lower pressure $P_{1}$ may be performed linearly:

$$
\sigma_{i}=\sigma_{i}\left(P_{1}\right)+m\left(P-P_{1}\right)
$$

where:

$$
m=\frac{\sigma_{i}\left(P_{2}\right)-\sigma_{i}\left(P_{1}\right)}{P_{2}-P_{1}}
$$

This results in interpolation residuals below $2.6 \%$ for the region $6000-30,000$ $\mathrm{cm}^{-1}$ for all pressures. However, below $6000 \mathrm{~cm}^{-1}$ for low pressures and below $1000 \mathrm{~cm}^{-1}$ for high pressures, there are spikes in interpolation residual (> $10 \%$ ) for individual wavenumber bins, notably in the wings of strong features. The wings of strong lines add appreciable amounts of opacity to wavenumber bins where there previously was negligible opacity in an non-linear fashion. In reality this effects only a small fraction of the bins $(\sim 0.7 \%)$ and changes the total cross section in the region $10-6000 \mathrm{~cm}^{-1}$ by less than $0.002 \%$ at worst, hence this should have negligible effect on practical applications.

\section{Conclusion}

$\mathrm{H}_{2}{ }^{16} \mathrm{O}$ line widths pressure-broadened by hydrogen and helium were calculated using the the averaged energy difference method and $J^{\prime} J^{\prime \prime}$-dependence 
technique. Rotational quantum numbers $J$ up to 50 were considered. The temperature dependence of the widths was derived from calculations made at temperatures in the range 300 - $2000 \mathrm{~K}$. The calculated data are in reasonable agreement with available experiment.

The widths and temperature exponents are presented in new ExoMol format as dataset exclusive . broad files and can be used to generate a temperature and pressure dependent Lorentzian-half width for every line of the BT2 line list, or any line list with at least $J^{\prime}-J^{\prime \prime}$ assignments.

High resolution pressure dependent absorption cross sections for $\mathrm{H}_{2}{ }^{16} \mathrm{O}$ have been calculated for a mixed $\mathrm{H}_{2} / \mathrm{He}$ (85/15\% by number) environment and a range of temperatures $(\mathrm{T}=300-2000 \mathrm{~K})$ and pressures $(\mathrm{P}=0.001$ 10 bar) relevant to exoplanet and cool star atmospheres. The static cross sections are available from the ExoMol website (www.exomol.com/data/molecules/H2O/1H216O/BT2/).

It is out intention to make the cross sections available through the ExoMol cross section service, a web-based interface (www.exomol.com/data/datatypes/xsec/) which currently allows astronomers to download zero pressure cross sections for available molecules at user defined temperatures and spectral resolution.

The form chosen for representing the pressure-broadening parameters is based on the quantum numbers of the upper and lower levels. This means that the parameters are transferable to other extensive water line lists such as new, complete and more accurate POKAZATEL line list [73] which will be released soon. Furthermore, the differences in pressure effects between $\mathrm{H}_{2}{ }^{16} \mathrm{O}$, and $\mathrm{H}_{2}{ }^{17} \mathrm{O}$ and $\mathrm{H}_{2}{ }^{18} \mathrm{O}$ should be very small. This means that the 
broadening files presented here should also be appropriated for the newly computed $\mathrm{H}_{2}{ }^{17} \mathrm{O}$ and $\mathrm{H}_{2}{ }^{18} \mathrm{O}$ hot line lists [74]

Pressure dependent absorption cross sections for other key species in the atmospheres of exoplanets and cool stars (for example $\mathrm{NH}_{3}, \mathrm{CO}, \mathrm{CO}_{2}$ and $\mathrm{CH}_{4}$ ) will also be included here in due course [75].

\section{Acknowledgements}

This work was supported by a grant from Energinet.dk project N. 20131-1027, by UCL through the Impact Studentship Program and the European Research Council under Advanced Investigator Project 267219 and partly supported by CNRS in the frame of the International Associated Laboratory SAMIA and RFBR No. 16-32-00244.

\section{References}

[1] J. Tennyson, S. N. Yurchenko, ExoMol: molecular line lists for exoplanet and other atmospheres, Mon. Not. R. Astron. Soc. 425 (2012) 21-33.

[2] G. Tinetti, J. Tennyson, C. A. Griffiths, I. Waldmann, Water in Exoplanets, Phil. Trans. Royal Soc. London A 370 (2012) 2749-2764.

[3] J. Tennyson, S. N. Yurchenko, A. F. Al-Refaie, E. J. Barton, K. L. Chubb, P. A. Coles, S. Diamantopoulou, M. N. Gorman, C. Hill, A. Z. Lam, L. Lodi, L. K. McKemmish, Y. Na, A. Owens, O. L. Polyansky, T. Rivlin, C. Sousa-Silva, D. S. Underwood, A. Yachmenev, E. Zak, The ExoMol database: molecular line lists for exoplanet and other hot 
atmospheres, J. Mol. Spectrosc. 327 (2016) 73-94. doi:10.1016/j·jms . 2016.05 .002 .

[4] G. Tinetti, T. Encrenaz, A. Coustenis, Spectroscopy of planetary atmospheres in our galaxy, Astron. Astrophys. Rev. 21 (2013) 1-65. doi:10.1007/s00159-013-0063-6.

[5] I. J. M. Crossfield, Observations of Exoplanet Atmospheres, Publ. Astron. Soc. Pac. 127 (2015) 956.

[6] G. Tinetti, A. Vidal-Madjar, M.-C. Liang, J.-P. Beaulieu, Y. Yung, S. Carey, R. J. Barber, J. Tennyson, I. Ribas, N. Allard, G. E. Ballester, D. K. Sing, F. Selsis, Water vapour in the atmosphere of a transiting extrasolar planet, Nature 448 (2007) 169-171.

[7] M. R. Swain, P. Deroo, C. A. Griffith, G. Tinetti, A. Thatte, G. Vasisht, P. Chen, J. Bouwman, I. J. Crossfield, D. Angerhausen, C. Afonso, T. Henning, A ground-based near-infrared emission spectrum of the exoplanet HD 189733b, Nature 463 (2010) 637-639.

[8] M. R. Swain, G. Vasisht, G. Tinetti, J. Bouwman, P. Chen, Y. Yung, D. Deming, P. Deroo, Molecular signatures in the near-infrared dayside spectrum of HD 189733b, Astrophys. J. 690 (2009) L114-L117. doi: 10.1088/0004-637X/690/2/L114.

[9] J. L. Birkby, R. J. de Kok, M. Brogi, E. J. W. de Mooij, H. Schwarz, S. Albrecht, I. A. G. Snellen, Detection of water absorption in the day side atmosphere of HD 189733 b using ground-based high-resolution 
spectroscopy at $3.2 \mathrm{mu}$ m, Mon. Not. R. Astron. Soc. 436 (2013) L35L39. doi:\{10.1093/mnrasl/slt107\}.

[10] P. R. McCullough, N. Crouzet, D. Deming, N. Madhusudhan, WATER VAPOR IN THE SPECTRUM OF THE EXTRASOLAR PLANET HD 189733b. I. THE TRANSIT, Astrophys. J. 791 (2014) 55. doi:\{10. $1088 / 0004-637 X / 791 / 1 / 55\}$.

[11] K. O. Todorov, D. Deming, A. Burrows, C. J. Grillmair, UPDATED SPITZER EMISSION SPECTROSCOPY OF BRIGHT TRANSITING HOT JUPITER HD 189733b, Astrophys. J. 796 (2014) 100. doi:\{10. 1088/0004-637X/796/2/100\}.

[12] K. B. Stevenson, J. Harrington, S. Nymeyer, N. Madhusudhan, S. Seager, W. C. Bowman, R. A. Hardy, D. Deming, E. Rauscher, N. B. Lust, Possible thermochemical disequilibrium in the atmosphere of the exoplanet GJ 436b, Nature 464 (2010) 1161-1164.

[13] H. A. Knutson, N. Madhusudhan, N. B. Cowan, J. L. Christiansen, E. Agol, D. Deming, J.-M. Désert, D. Charbonneau, G. W. Henry, D. Homeier, J. Langton, G. Laughlin, S. Seager, A Spitzer transmission spectrum for the exoplanet GJ 436b, evidence for stellar variability, and constraints on dayside flux variations, Astrophys. J. 735 (2011) 27. doi:10.1088/0004-637X/735/1/27.

[14] J. P. Beaulieu, D. M. Kipping, V. Batista, G. Tinetti, I. Ribas, S. C. J. A. Noriega-Crespo, C. A. Griffith, G. Campanella, S. Dong, J. Tennyson, R. J. Barber, P. Deroo, S. J. Fossey, D. Liang, M. R. Swain, Y. Yung, 
Water in HD 209458b's atmosphere from $3.6-8 \mu \mathrm{m}$ IRAC photometric observations in primary transit, Mon. Not. R. Astron. Soc. 409 (2010) 963-974.

[15] D. Deming, A. Wilkins, P. McCullough, A. Burrows, J. J. Fortney, E. Agol, I. Dobbs-Dixon, N. Madhusudhan, N. Crouzet, J.-M. Desert, R. L. Gilliland, K. Haynes, H. A. Knutson, M. Line, Z. Magic, A. M. Mandell, S. Ranjan, D. Charbonneau, M. Clampin, S. Seager, A. P. Showman, Infrared transmission spectroscopy of the exoplanets HD 209458b and XO-1b using the wide field camera-3 on the hubble space telescope, Astrophys. J. 774 (2013) 95. doi:10.1088/0004-637X/774/ $2 / 95$.

[16] G. Tinetti, P. Deroo, M. R. Swain, C. A. Griffith, G. Vasisht, L. R. Brown, C. Burke, P. McCullough, Probing the terminator region atmosphere of the hot-jupiter xo-1b with transmission spectroscopy, Astrophys. J. Lett. 712 (2010) L139-L142. doi:10.1088/2041-8205/712/2/ L139.

[17] H. R. Wakeford, D. K. Sing, D. Deming, N. P. Gibson, J. J. Fortney, A. S. Burrows, G. Ballester, N. Nikolov, S. Aigrain, G. Henry, H. Knutson, A. L. des Etangs, F. Pont, A. P. Showman, A. Vidal-Madjar, K. Zahnle, HST hot Jupiter transmission spectral survey: detection of water in HAT-P-1b from WFC3 near-IR spatial scan observations, Mon. Not. R. Astron. Soc. 435 (2013) 3481-3493. doi:10.1093/mnras/stt1536.

[18] M. Brogi, R. J. de Kok, J. L. Birkby, H. Schwarz, I. A. G. Snellen, Carbon monoxide and water vapor in the atmosphere of the non- 
transiting exoplanet HD 179949 b, Astron. Astrophys. 565 (2014) A124. doi : 10.1051/0004-6361/201423537.

[19] C. M. Huitson, D. K. Sing, F. Pont, J. J. Fortney, A. S. Burrows, P. A. Wilson, G. E. Ballester, N. Nikolov, N. P. Gibson, D. Deming, S. Aigrain, T. M. Evans, G. W. Henry, A. L. des Etangs, A. P. Showman, A. VidalMadjar, K. Zahnle, An HST optical-to-near-IR transmission spectrum of the hot Jupiter WASP-19b: detection of atmospheric water and likely absence of TiO, Mon. Not. R. Astron. Soc. 434 (2013) 3252-3274. doi: $\{10.1093 / \mathrm{mnras} / \mathrm{stt} 1243\}$.

[20] L. Kreidberg, M. R. Line, J. L. Bean, K. B. Stevenson, J.-M. Desert, N. Madhusudhan, J. J. Fortney, J. K. Barstow, G. W. Henry, M. H. Williamson, A. P. Showman, A DETECTION OF WATER IN THE TRANSMISSION SPECTRUM OF THE HOT JUPITER WASP-12b AND IMPLICATIONS FOR ITS ATMOSPHERIC COMPOSITION, Astrophys. J. 814 (2015) 66. doi:\{10.1088/0004-637X/814/1/66\}.

[21] Q. M. Konopacky, T. S. Barman, B. A. Macintosh, C. Marois, Detection of Carbon Monoxide and Water Absorption Lines in an Exoplanet Atmosphere, Science 339 (2013) 1398-1401. doi:10.1126/science.1232003.

[22] T. S. Barman, Q. M. Konopacky, B. Macintosh, C. Marois, Simultaneous detection of water, methane, and carbon monoxide in the atmosphere of exoplanet hr 8799 b, Astrophys. J. 804 (2015) 61. doi:10.1088/ 0004-637X/804/1/61.

[23] C. M. Sharp, A. Burrows, Atomic and molecular opacities for brown 
dwarf and giant planet atmospheres, Astrophys. J. Suppl. 168 (2007) 140.

[24] P. F. Bernath, Molecular astronomy of cool stars and sub-stellar objects, Int. Rev. Phys. Chem. 28 (2009) 681-709. doi:10.1080/ 01442350903292442 .

[25] F. Allard, D. Homeier, B. Freytag, Models of very-low-mass stars, brown dwarfs and exoplanets, Phil. Trans. Royal Soc. London A 370 (2012) 2765-2777. doi:\{10.1098/rsta.2011.0269\}.

[26] C. V. Morley, M. S. Marley, J. J. Fortney, R. Lupu, D. Saumon, T. Greene, K. Lodders, Water clouds in y dwarfs and exoplanets, Astrophys. J. 787 (2014) 78. doi:10.1088/0004-637X/787/1/78.

[27] F. Allard, P. H. Hauschildt, S. Miller, J. Tennyson, The influence of $\mathrm{H}_{2} \mathrm{O}$ line blanketing on the spectra of cool dwarf stars, Astrophys. J. 426 (1994) L39-L41.

[28] J. K. Faherty, C. G. Tinney, A. Skemer, A. J. Monson, Indications of Water Clouds in the Coldest Known Brown Dwarf, Astrophys. J. 793 (2014) L16.

[29] A. J. Skemer, C. V. Morley, K. N. Allers, T. R. Geballe, M. S. Marley, J. J. Fortney, J. K. Faherty, G. L. Bjoraker, R. Lupu, THE FIRST SPECTRUM OF THE COLDEST BROWN DWARF, Astrophys. J. Lett. 826 (2016) L17. doi:\{10.3847/2041-8205/826/2/L17\}.

[30] L. S. Rothman, I. E. Gordon, R. J. Barber, H. Dothe, R. R. Gamache, A. Goldman, V. I. Perevalov, S. A. Tashkun, J. Tennyson, HITEMP, the 
High-Temperature Molecular Spectroscopic Database, J. Quant. Spectrosc. Radiat. Transf. 111 (2010) 2139-2150.

[31] I. E. Gordon, L. S. Rothman, R. R. Gamache, D. Jacquemart, C. Boone, P. F. Bernath, M. W. Shephard, J. S. Delamere, S. A. Clough, Current updates of the water-vapor line list in HITRAN: A new "diet" for airbroadened half-widths, J. Quant. Spectrosc. Radiat. Transf. 108 (2007) 389-402. doi:10.1016/j.jqsrt.2007.06.009.

[32] R. J. Barber, J. Tennyson, G. J. Harris, R. N. Tolchenov, A high accuracy computed water line list, Mon. Not. R. Astron. Soc. 368 (2006) 1087-1094.

[33] C. Hill, S. N. Yurchenko, J. Tennyson, Temperature-dependent molecular absorption cross sections for exoplanets and other atmospheres, Icarus 226 (2013) 1673-1677.

[34] L. R. Brown, C. Plymate, $\mathrm{H}_{2}$-broadened $\mathrm{H}_{2}{ }^{16} \mathrm{O}$ in four infrared bands between 55 and $4045 \mathrm{~cm}^{-1}$, J. Quant. Spectrosc. Radiat. Transf. 56 (1996) 263 - 282. doi:10.1016/0022-4073(95)00191-3.

[35] D. W. Steyert, W. Wang, J. Sirota, N. M. Donahue, D. C. Reuter, Hydrogen and helium pressure broadening of water transitions in the 380-600 $\mathrm{cm}^{-1}$ region, J. Quant. Spectrosc. Radiat. Transf. 83 (2004) 183-191. doi:10.1016/S0022-4073(02)00300-X.

[36] L. R. Brown, D. C. Benner, V. M. Devi, M. A. H. Smith, R. A. Toth, Line mixing in self- and foreign-broadened water vapor at 6 microns, J. Mol. Struct. 742 (2005) 111-122. 
[37] Y. G. Golubiatnikov, Shifting and broadening parameters of the water vapor 183-GHz line $\left(3_{13}-2_{20}\right)$ by $\mathrm{H}_{2} \mathrm{O}, \mathrm{O}_{2}, \mathrm{~N}_{2}, \mathrm{CO}_{2}, \mathrm{H}_{2}, \mathrm{He}, \mathrm{Ne}$, Ar, and Kr at room temperature, J. Mol. Spectrosc. 230 (2005) 196-198.

[38] A. Lucchesinia, S. Gozzini, C. Gabbanini, Water vapor overtones pressure line broadening and shifting measurements, Eur. Phys. J. D 8 (2000) $223-226$.

[39] V. Zeninari, B. Parvitte, D. Courtois, N. N. Lavrentieva, Y. N. Ponomarev, G. Durry, Pressure broadening and shift coefficients of $\mathrm{H}_{2} \mathrm{O}$ due to perturbation by $\mathrm{N}_{2}, \mathrm{O}_{2}, \mathrm{H}_{2}$ and $\mathrm{He}$ in the 1.39 micron region: experiment and calculations, Mol. Phys. 102 (2004) 1697-1706.

[40] M. J. Dick, B. J. Drouin, J. C. Pearson, A collisional cooling investigation of the pressure broadening of the $1_{10}-1_{01}$ transition of water from 17 to 200 K, J. Quant. Spectrosc. Radiat. Transf. 110 (2009) 619 - 627. doi:10.1016/j.jqsrt.2008.11.012.

[41] S. Langlois, T. P. Birbeck, R. K. Hanson, Temperature-dependent collision-broadening parameters of $\mathrm{h}_{2} \mathrm{O}$ lines in the $1.4 \mu \mathrm{m}$ region using diode laser absorption spectroscopy, J. Mol. Spectrosc. 167 (1994) 272 - 281. doi:http://dx.doi.org/10.1006/jmsp.1994.1234.

[42] J. M. Dutta, C. R. Jones, T. M. Goyette, F. C. Lucia, The Hydrogen and Helium Pressure Broadening at Planetary Temperatures of the 183 and $380 \mathrm{GHz}$ Transitions of Water Vapor, Icarus 102 (1993) 232-239.

[43] R. R. Gamache, R. Lynch, L. R. Brown, Theoretical calculations of pressure broadening coefficients for $\mathrm{H}_{2} \mathrm{O}$ perturbed by hydrogen or he- 
lium gas, J. Quant. Spectrosc. Radiat. Transf. 56 (1996) $471-487$. doi:10.1016/0022-4073(96)00098-2.

[44] A. Faure, L. Wiesenfeld, J. Tennyson, B. J. Drouin, Pressure broadening of water and carbon monoxide transitions by molecular hydrogen at high temperatures, J. Quant. Spectrosc. Radiat. Transf. 116 (2013) 79-86.

[45] B. Drouin, L. Wiesenfeld, Low-temperature waterhydrogen-molecule collisions probed by pressure broadening and line shift, Phys. Rev. A 86 (2012) 022705.

[46] T. M. Petrova, A. M. Solodov, A. A. Solodov, V. I. Starikov, Measurements and calculations of He-broadening and -shifting parameters of the transitions of the $\nu_{1}+\nu_{2}+\nu_{3}$ bands., Mol. Phys. 110 (2012) 1493-1503.

[47] T. M. Petrova, A. M. Solodov, A. A. Solodov, V. I. Starikov, Vibrational dependence of an intermolecular potential for $\mathrm{H}_{2} \mathrm{O}$-He system, J. Quant. Spectrosc. Radiat. Transf. 129 (2013) 241-253.

[48] T. M. Petrova, A. M. Solodov, A. A. Solodov, V. I. Starikov, Broadening parameters of the $\mathrm{H}_{2} \mathrm{O}-\mathrm{He}$ collisional system for astrophysical applications, J. Mol. Spectrosc. 321 (2016) 50-58.

[49] A. M. Solodov, V. I. Starikov, Broadening and shift lines of the $\nu_{2}+\nu_{3}$ band of water vapour induced by helium pressure., Opt. Spectrosc. 105 (2008) 14-20.

[50] A. M. Solodov, V. I. Starikov, Helium-induced halfwidths and line shifts of water vapour transitions of the $\nu_{1}+\nu_{2}$ and $\nu_{2}+\nu_{3}$ bands., Mol. Phys. 104 (2009) 43-51. 
[51] T. M. Goyette, F. C. D. Lucia, The pressure broadening of the $3_{13}-2_{20}$ transition of water between 80 - 600k, J. Mol. Spectrosc. 143 (1990) 346-358.

[52] M. Godon, A. Bauer, Helium-broadened widths of the 183 and $380 \mathrm{GHz}$ lines of water vapour, Chem. Phys. Lett. 147 (1988) 189-191.

[53] V. V. Lazarev, Y. N. Ponomarev, B. Sumpf, O. Fleischmann, J. Waschull, H. Kronfeldt, V. N. Stroinova, Noble Gas Pressure-Induced Broadening and Shift of $\mathrm{H}_{2} \mathrm{O} \mathrm{SO} \mathrm{S}_{2}$ Absorption Lines, J. Mol. Spectrosc. 173 (1995) 177-193.

[54] P. Poddar, S. Mitra, M. M. Hossain, D. Biswas, P. N. Ghosh, B. Ray, Diode laser spectroscopy of $\mathrm{He}, \mathrm{N}_{2}$ and air broadened water vapour transitions belonging to the $2 \nu_{1}+\nu_{2}+\nu_{3}$ overtone band, Mol. Phys. 108 (2010) 1957-1964.

[55] C. Claveau, A. Henry, D. Hurtmans, A. Valentin, Narrowing and broadening parameters of $\mathrm{H}_{2} \mathrm{O}$ lines perturbed by $\mathrm{He}, \mathrm{Ne}, \mathrm{Ar}, \mathrm{Kr}$ and nitrogen in the spectral range $1850-2140 \mathrm{~cm}^{-1}$, J. Quant. Spectrosc. Radiat. Transf. 68 (2001) 273-298.

[56] C. Claveau, A. Valentin, Narrowing and broadening parameters of $\mathrm{H}_{2} \mathrm{O}$ lines perturbed by Helium, argon and xenon in the 1170-1440 $\mathrm{cm}^{-1}$ spectral range, Mol. Phys. 107 (2009) 1417-1422.

[57] R. H. Dicke, The Effect of Collisions upon the Doppler Width of Spectral Lines., Phys. Rev. 86 (1953) 472-473. 
[58] J. P. Wittke, R. H. Dicke, Redetermination of the Hyperfine Splitting in the Ground State of Atomic Hydrogen., Phys. Rev. 103 (1956) 620-631.

[59] N. N. Lavrentieva, A. S. Dudaryonok, Q. Ma, Line broadening estimate from averaged energy differences of coupled states., Proceedings of SPIE. 92920M (2014) 1-9.

[60] B. A. Voronin, N. N. Lavrentieva, T. P. Mishina, T. Y. Chesnokova, M. J. Barber, J. Tennyson, Estimate of the $J^{\prime} J^{\prime \prime}$ dependence of water vapour line broadening parameters, J. Quant. Spectrosc. Radiat. Transf. 111 (2010) 2308-2314.

[61] Q. Ma, R. H. Tipping, C. Boulet, Modification of the Robert-Bonamy formalism in calculating Lorentzian half-widths and shifts., J. Quant. Spectrosc. Radiat. Transf. 103 (2007) 588-596.

[62] B. K. Anthony, P. R. Gamache, C. D. Szembek, D. N. Niles, R. R. Gamache, Modified complex Robert-Bonamy formalism calculations for strong to weak interacting systems., Mol. Phys. 104 (2006) 1-9.

[63] R. Lynch, R. R. Gamache, S. P. Neshyba, $\mathrm{N}_{2}$ and $\mathrm{O}_{2}$ induced half-widths and line shifts of water vapour lines in the (301) - (000) and (221) - (000), J. Quant. Spectrosc. Radiat. Transf. 59 (1998) 595.

[64] R. R. Gamache, R. Lynch, S. P. Neshyba, New developments in the theory of pressure-broadening and presure-shifting of spectral lines of $\mathrm{H}_{2} \mathrm{O}$ : The complex Robert-Bonamy formalism., J. Quant. Spectrosc. Radiat. Transf. 59 (1998) 319. 
[65] R. Lynch, Half-widths and line shifts of water vapour perturbed by both nirtogen and oxygen., Ph.D. thesis, Physics Department, University of Massachusetts Lowell (1995).

[66] J. T. Hodges, D. Lisak, N. Lavrentieva, A. Bykov, L. Sinitsa, J. Tennyson, R. J. Barber, R. N. Tolchenov, Comparison between theoretical calculations and high-resolution measurements of pressure-broadening for water vapor spectra near 935 nm, J. Mol. Spectrosc. 249 (2008) 8694.

[67] M. S. Child, T. Weston, J. Tennyson, Quantum monodromy in the spectrum of $\mathrm{H}_{2} \mathrm{O}$ and other systems: new insight into the level structures of quasi-linear molecules, Mol. Phys. 96 (1999) 371-379.

[68] J. S. Wilzewski, I. E. Gordon, R. V. Kochanov, C. Hill, L. S. Rothman, $\mathrm{H}_{2}$, He, and $\mathrm{CO}_{2}$ line-broadening coefficients, pressure shifts and temperature-dependence exponents for the HITRAN database. Part 1: $\mathrm{SO}_{2}, \mathrm{NH}_{3}, \mathrm{HF}, \mathrm{HCl}, \mathrm{OCS}$ and $\mathrm{C}_{2} \mathrm{H}_{2}$, J. Quant. Spectrosc. Radiat. Transf. 168 (2016) 193 - 206. doi:10.1016/j.jqsrt.2015.09.003.

[69] J. Tennyson, P. F. Bernath, A. Campargue, A. G. Császár, L. Daumont, R. R. Gamache, J. T. Hodges, D. Lisak, O. V. Naumenko, L. S. Rothman, H. Tran, N. F. Zobov, J. Buldyreva, C. D. Boone, M. D. De Vizia, L. Gianfrani, J.-M. Hartmann, R. McPheat, J. Murray, N. H. Ngo, O. L. Polyansky, D. Weidmann, Recommended isolated-line profile for representing high-resolution spectroscopic transitions (IUPAC Technical Report), Pure Appl. Chem. 86 (2014) 1931-1943. 
[70] S. G. Johnson, The Faddeeva package, http://abinitio.mit.edu/wiki/index.php/Faddeeva_Package (2012).

[71] M. Vidler, J. Tennyson, Accurate partition function and thermodynamic data for water, J. Chem. Phys. 113 (2000) 9766-9771.

[72] C. Hedges, N. Madhusudhan, Effect of pressure broadening on molecular absorption cross sections in exoplanetary atmospheres, Mon. Not. R. Astron. Soc. 458 (2) (2016) 1427-1449. doi:10.1093/mnras/stw278.

[73] O. L. Polyansky, A. A. Kyuberis, L. Lodi, J. Tennyson, R. I. Ovsyannikov, N. Zobov, S. N. Yurchenko, ExoMol molecular line lists XX: high accuracy computed line list for hot $\mathrm{H}_{2}{ }^{16} \mathrm{O}$, Mon. Not. R. Astron. Soc.

[74] O. L. Polyansky, A. A. Kyuberis, L. Lodi, J. Tennyson, R. I. Ovsyannikov, N. Zobov, ExoMol molecular line lists XIX: high accuracy computed line lists for $\mathrm{H}_{2}{ }^{17} \mathrm{O}$ and $\mathrm{H}_{2}{ }^{18} \mathrm{O}$, Mon. Not. R. Astron. Soc.

[75] E. J. Barton, C. Hill, M. Czurylo, H.-Y. Li, A. Hyslop, S. N. Yurchenko, J. Tennyson, The ExoMol diet of line-by-line pressure-broadening parameters, J. Quant. Spectrosc. Radiat. Transf. 